Draft version August 20, 2018

Preprint typeset using $\mathrm{LAT}_{\mathrm{E}} \mathrm{X}$ style emulateapj v. 12/01/06

\title{
STAR FORMATION AND THE INTERSTELLAR MEDIUM IN $Z>6$ UV-LUMINOUS LYMAN-BREAK GALAXIES
}

Chris J. Willott

NRC Herzberg, 5071 West Saanich Rd, Victoria, BC V9E 2E7, Canada

Chris L. Carilli

National Radio Astronomy Observatory, P.O. Box 0, Socorro, NM 87801, USA and Cavendish Astrophysics Group, University of Cambridge, Cambridge, CB3 0HE, UK

JEFF WAGG

Square Kilometre Array Organization, Jodrell Bank Observatory, Lower Withington, Macclesfield, Cheshire SK11 9DL, UK

RAN WANG

Kavli Institute for Astronomy and Astrophysics, Peking University, Beijing 100871, China

Draft version August 20, 2018

\begin{abstract}
We present Atacama Large Millimeter Array (ALMA) detections of atomic carbon line and dust continuum emission in two UV-luminous galaxies at redshift 6. The far-infrared (FIR) luminosities of these galaxies are substantially lower than similar starbursts at later cosmic epochs, indicating an evolution in the dust properties with redshift, in agreement with the evolution seen in ultraviolet (UV) attenuation by dust. The [C II] to FIR ratios are found to be higher than at low redshift showing that $[\mathrm{C}$ II] should be readily detectable by ALMA within the reionization epoch. One of the two galaxies shows a complex merger nature with the less massive component dominating the UV emission and the more massive component dominating the FIR line and continuum. Using the interstellar atomic carbon line to derive the systemic redshifts we investigate the velocity of Ly $\alpha$ emission emerging from high- $z$ galaxies. In contrast to previous work, we find no evidence for decreasing Ly $\alpha$ velocity shifts at high-redshift. We observe an increase in velocity shifts from $z \approx 2$ to $z \approx 6$, consistent with the effects of increased IGM absorption.
\end{abstract}

Subject headings: cosmology: observations - galaxies: evolution — galaxies: formation — galaxies: high-redshift

\section{INTRODUCTION}

Observations from the Hubble Space Telescope have given us a broad-brush picture of the evolution of galaxies over cosmic time. Galaxies in the reionization epoch at redshifts $z>6$ were rarer, smaller, bluer and had stronger nebular emission lines than typical galaxies in the more evolved universe (Ono et al. 2013, Bouwens et al. 2014b a; Smit et al. 2014). These observations can be explained by a Lambda Cold Dark Matter cosmology with initial generations of stars forming out of low metallicity gas in relatively low mass halos. However, many details are still to be resolved.

Whether the early galaxy population is able to generate enough photons to complete cosmic reionization by redshift 6 is still a matter of debate and depends upon uncertainties such as the clumpiness of the intergalactic medium (IGM) (Finlator et al. 2012), the escape fraction of ionizing photons from galaxies (Siana et al. 2015) and the UV spectra of very low-mass galaxies (Schaerer \& de Barros 2010). Strong evolution in the fraction of galaxies showing Ly $\alpha$ emission (Pentericci et al. 2011; Schenker et al. 2012; Treu et al. 2013) suggests a rapid change in the IGM neutral hydrogen fraction at $z \sim 7$. Some of the strength of this evolution could also be accounted

Electronic address: chris.willott@nrc.ca for by increased Ly $\alpha$ absorption within galaxies due to lower Ly $\alpha$ velocity shifts (Choudhury et al. 2014). The first measurements of Ly $\alpha$ velocity shifts in two $z>6$ galaxies do indeed appear to show smaller shifts than at lower redshift (Stark et al. 2014a).

Up til now most of our knowledge of high-redshift galaxies is based on rest-frame ultraviolet (UV) and optical observations. Observations in the far-infrared (FIR) can provide information on the physical conditions of the interstellar medium (ISM) and dust and molecular gas in obscured star-forming regions, as well as gas kinematics. At $z \sim 2$ FIR photometry of rest-frame UV-selected galaxies shows that the star formation rate (SFR) is underestimated by a factor of 5 from UV measurements alone (Reddy et al. 2012). With the incredible sensitivity of the Atacama Large Millimeter Array (ALMA) it should be possible to detect dust continuum emission, molecular and atomic gas in high-redshift galaxies to complete our understanding of their physical properties (Carilli \& Walter 2013). In addition, the line data provides information on the gas kinematics and can be used to derive dynamical masses, separate spatially unresolved mergers and define a systemic redshift against which to measure the emergent Ly $\alpha$ kinematics.

Several studies using ALMA and other facilities have resulted in non-detections and claims that the FIR line 
and/or continuum fluxes are lower than expected based on UV SFRs (Walter et al. 2012; Kanekar et al. 2013. Ouchi et al. 2013; Ota et al. 2014, González-López et al. 2014 Maiolino et al. 2015; Schaerer et al. 2015). ALMA observations failed to detect either $1.2 \mathrm{~mm}$ continuum or the fine-structure line of singly-ionized carbon, [C II], in the $z>6.5$ galaxies Himiko and IOK-1 (Ouchi et al. 2013 Ota et al. 2014), despite the strong UV continuum indicating $\mathrm{SFR} \sim 20-100 M_{\odot} \mathrm{yr}^{-1}$. They showed that the FIR (cool dust) contributions to the spectral energy distributions are similar to nearby dwarf irregulars, more than an order of magnitude below the levels expected for nearby starbursts/spirals such as M82 and M51. The physical interpretation of the lack of dust and [C II] emission in these $z>6$ galaxies is a very low metallicity ISM. Observations of local low metallicity star-forming galaxies such as I Zw 18 also show very low dust-to-gas and dust-to-stars ratios, further confirming such galaxies as analogues of high- $z$ galaxies (Fisher et al. 2014).

This picture has brightened recently with several detections of the ISM in distant galaxies. Although Maiolino et al. (2015) failed to detect three $6.8<z<7.1$ Lyman Break Galaxies (LBGs) with ALMA in line or continuum, they did detect [C II] emission at the expected redshift of BDF3299, offset by $4 \mathrm{kpc}$ from the UV position, which they interpret as an accreting or satellite clump of gas. Watson et al. (2015) detected a gravitationallylensed galaxy at $z=7.5$ in dust continuum from which they infer $S F R_{\mathrm{FIR}}$ about 3 times greater than $S F R_{\mathrm{UV}}$, similar to the ratio typically observed at $z \sim 2$ (Reddy et al. 2012). The lack of a [C II] detection in this galaxy is likely due to an uncertain redshift, as Watson et al. note the lack of an emission line redshift means the ALMA data only cover $50 \%$ of potential [C II] line frequencies. Capak et al. (2015) observed nine LBGs at $5<z<6$ with ALMA and detected [C II] emission from all of them and dust continuum in four galaxies. They showed that the dust emission in these galaxies is much weaker than expected based on similar galaxies at lower redshift. The Capak LBGs were selected based on interstellar UV lines so likely have higher metallicity than most previouslytargeted high- $z$ galaxies, although still lower than at low redshift.

We are carrying out an ALMA program targeting $U V$ luminous LBGs at $z>6$. In this paper we present observations of the first two galaxies observed from our sample. The galaxies were first identified in Canada-FranceHawaii Telescope (CFHT) optical and near-IR imaging and both have spectroscopic redshifts based on a continuum break plus Ly $\alpha$ emission. The galaxy which we name here as CLM 1 was discovered by Cuby et al. (2003) at a redshift of $z=6.17$. The other galaxy, WMH 5 at $z=6.07$, was discovered in Willott et al. (2013b). Both galaxies have near-IR continuum magnitudes $A B \sim 24$ making them among the brightest rest-frame UV galaxies known at this epoch.

In Section 2 we describe our observations and in Section 3 our results. Section 4 discusses the ratio of [C II] to FIR continuum and Section 5 the Ly $\alpha$ velocity shifts observed. We draw conclusions in Section 6. Cosmological parameters of $H_{0}=67.8 \mathrm{~km} \mathrm{~s}^{-1} \mathrm{Mpc}^{-1}, \Omega_{\mathrm{M}}=0.308$ and $\Omega_{\Lambda}=0.692$ (Planck Collaboration et al. 2015) are assumed throughout.

\section{OBSERVATIONS}

\subsection{ALMA}

Observations were made during ALMA cycle 2, in June 2014, with between 29 and 32 antennas, and a maximum baseline of $650 \mathrm{~m}$. A total bandwidth of $7.2 \mathrm{GHz}$ was employed in Band 6 using 4 dual-polarization sub-bands between 249 and $272 \mathrm{GHz}$. One of the sub-bands was centered at the [C II] line (rest frame $1900.5369 \mathrm{GHz}$ ) for each source. The rest of the bands were used for continuum measurements. A total of 95 minutes on-source integration time was obtained for each galaxy.

The initial data editing and calibration were performed as part of standard data processing by the ALMA staff. The calibrated visibility data were then re-analyzed, performing additional flagging of bad time periods and bad channels. The data were re-imaged using the CASA Briggs weighting of the visibility data with Robust $=1$ to create continuum and channel images. For both sources, the Gaussian restoring beam was close to circular, with a FWHM $=0.50 "$. Spectral cubes at $15.625 \mathrm{MHz}$ resolution $\left(17.5 \mathrm{~km} \mathrm{~s}^{-1}\right)$ were synthesized, and smoothed spectrally for subsequent analysis, as required. The continuum was subtracted in the image-plane, using the off-line channels in the line cube.

The spectra were transformed from the observed topocentric system to the local standard of rest (LSR). The velocity offsets applied were $\approx-11 \mathrm{~km} \mathrm{~s}^{-1}$.

Spectral and spatial Gaussian fitting was performed using the CASA viewer, CASA fitting tools and custom software. Results for total line fluxes, continuum flux densities, and parametric source sizes, are given below. Flux uncertainties have $10 \%$ added in quadrature for absolute flux calibration uncertainty.

\subsection{Near- and Mid-Infrared}

To compare with the ALMA data we use near-infrared (NIR) data from the ESO VISTA VIDEO survey (Jarvis et al. 2013). Images from Data Release 3 in bands $Z, Y, J, H$ and $K s$ were obtained, sampling the restframe ultraviolet continua. Astrometric calibration of the VIDEO images to the radio reference frame used by ALMA was performed by matching bright VIDEO sources to the AllWISE catalog (Cutri et al. 2013) and extracting the ALMA phase calibrator positions from the AllWISE catalog. Given the size of the residuals this process gives an uncertainty on astrometric frame matching of $\approx 00^{\prime \prime} 1$, comparable to the positional uncertainties of the NIR data due to $\mathrm{S} / \mathrm{N}$ and seeing. Fluxes in the five NIR bands of the two target galaxies were determined using aperture photometry with aperture corrections to total fluxes. Due to the similar shapes, fluxes and S/N of the data in the three bluest VIDEO bands, a deeper combined $Z Y J$ image of each field was generated. Neither galaxy is significantly spatially resolved in the NIR data.

The two galaxies have been observed with the Spitzer Space Telescope in the IRAC 3.6 and $4.5 \mu \mathrm{m}$ bands during the SERVS survey (Mauduit et al. 2012). Due to the proximity of bright galaxies along the line-of-sight to both $z>6$ galaxies it was necessary to perform simultaneous fitting of all objects in the IRAC field using the $Z Y J$ VIDEO image as a prior for the object shapes. Models were fit to the NIR images using GALFIT (Peng 


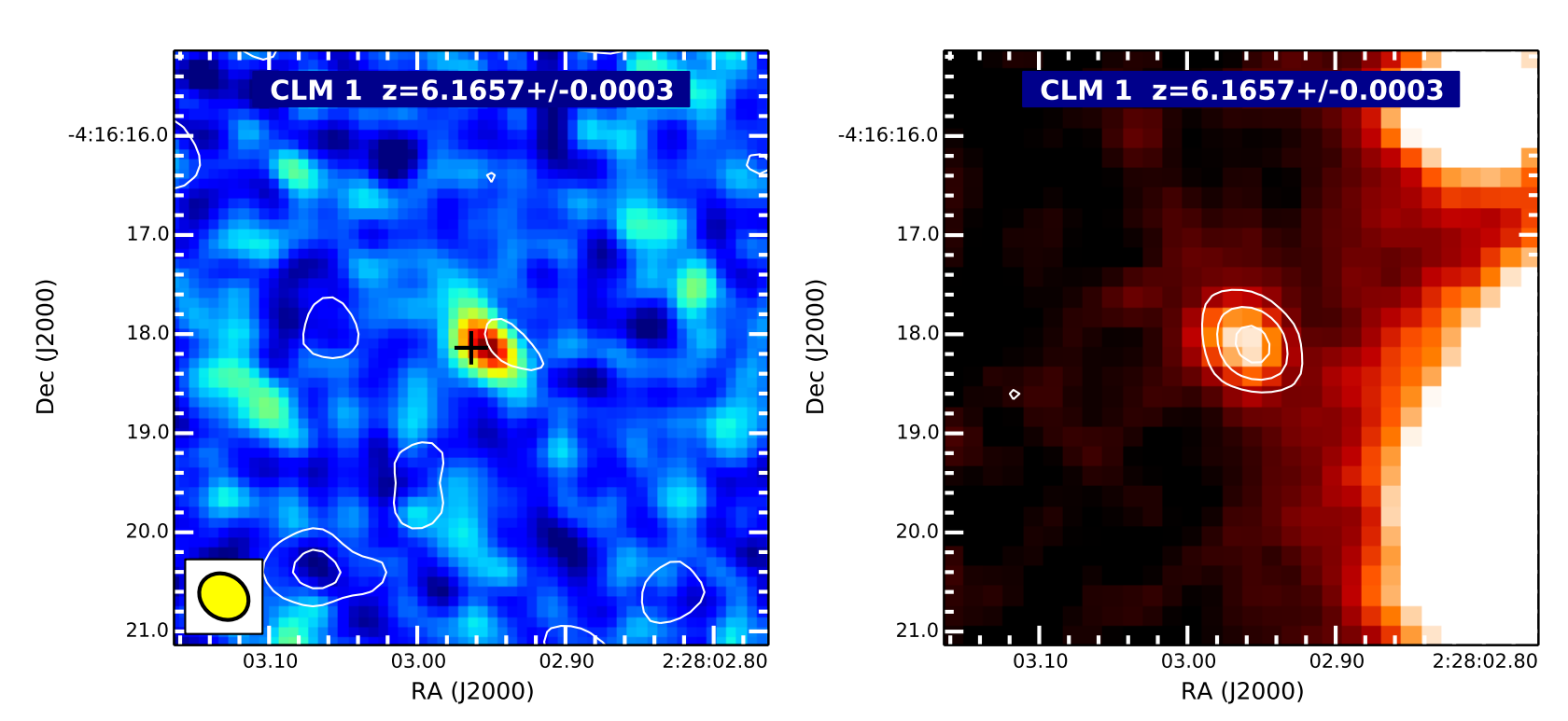

FIG. 1. - Left: The background image is ALMA integrated [C II] line map of CLM 1. White contours are the $1.2 \mathrm{~mm}$ continuum emission from the three line-free basebands at contour levels 1.5, $2.5 \sigma$ beam $^{-1}$, specially chosen to show the low $\mathrm{S} / \mathrm{N}$ possible continuum detection close to the $\mid \mathrm{C}$ II $\mid$ emission. The near-infrared centroid is plotted as a black plus symbol. The restoring beam is shown in yellow. Right: The background is the $z Y J$ NIR image. Contours show the ALMA [C II] emission from the left panel at levels $3,5,7 \sigma$ beam ${ }^{-1}$. The rest-frame UV continuum and [C II] emission are co-spatial. Two foreground galaxies are visible on the right side of the image.
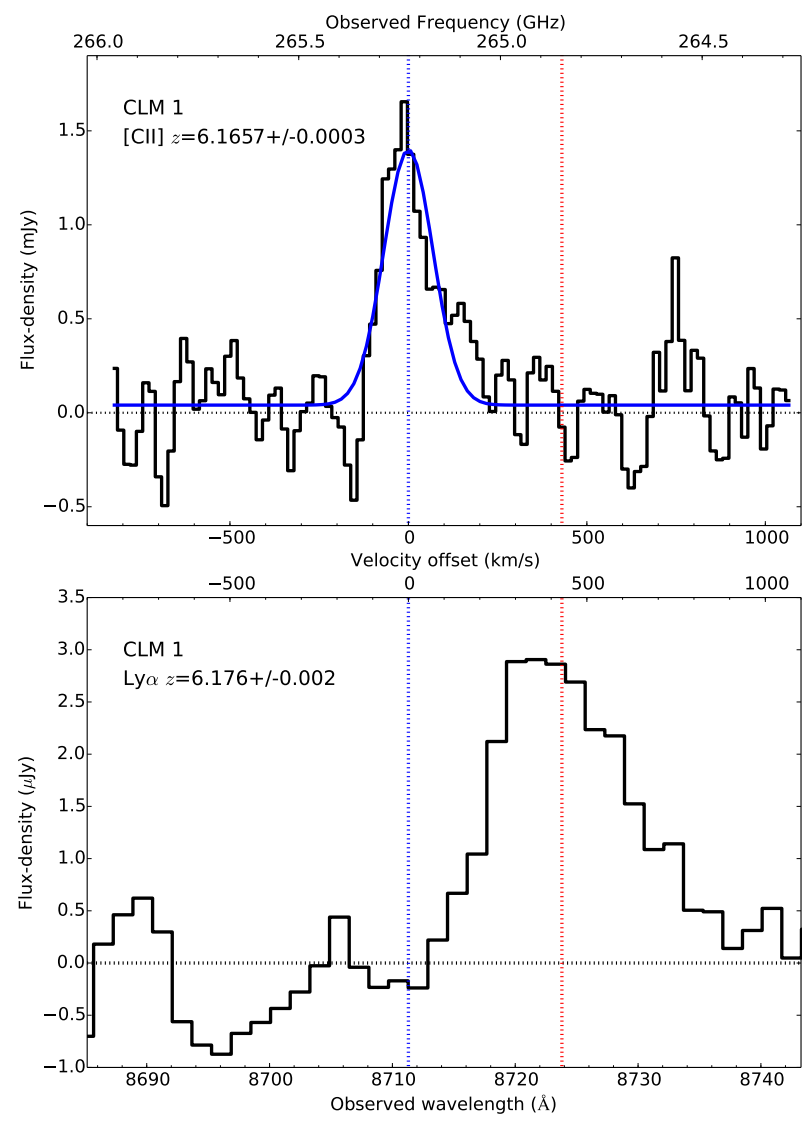

Fig. 2.- ALMA [C II] (upper) and VLT FORS2 Ly $\alpha$ (lower) spectra of CLM 1. The ALMA spectrum is well fit by a single Gaussian emission line, with a marginal red wing excess. The Lya spectrum shows typical blue-absorbed asymmetry. Vertical blue and red dotted lines mark the [C II] Gaussian fit peak and Lya flux-weighted centroid, respectively. Ly $\alpha$ is offset from [C II] by $+430 \pm 69 \mathrm{~km} \mathrm{~s}^{-1}$. et al. 2010) and subsequent fitting of the IRAC data was done with PyGFIT (Mancone et al. 2013). Both $z>6$ galaxies are clearly detected at both 3.6 and $4.5 \mu \mathrm{m}$, although with flux uncertainties of $\sim 30 \%$.

\subsection{Lyman- $\alpha$ spectroscopy}

Optical spectroscopy, including the detection of asymmetric Ly $\alpha$ emission lines and continuum breaks, of both galaxies were presented in their discovery papers (Cuby et al. 2003, Willott et al. 2013b). In order to compare these spectra to the ALMA spectra they were corrected from observed wavelength to the LSR by applying velocity corrections of $+26 \mathrm{~km} \mathrm{~s}^{-1}$ and $+32 \mathrm{~km} \mathrm{~s}^{-1}$ for CLM 1 and WMH 5, respectively. The spectrum of CLM 1 was obtained using FORS2 at the ESO VLT with a spectral resolution of $R=1400$ and that of WMH 5 using GMOS at Gemini-North with $R=1000$.

\section{RESULTS \\ 3.1. CLM 1}

For both galaxies there are strong detections of the [C II] line evident in the datacubes at close to the expected position and velocity. In Figure 1 we present the ALMA and NIR imaging of the galaxy CLM 1. The [C II] map is made from a sum over all channels showing significant line emission. The [C II] emission is spatially extended along a similar direction as the beam extension. A Gaussian fit to the source gives an observed size of $0.73 \times 0.42$ at position angle east of north $(\mathrm{PA})=$ 42 , compared with a beam size of $0 . .52 \times 0$. . 44 at $\mathrm{PA}=$ 55. The CASA fitting software does not provide a deconvolved source size due to the only slightly larger size than the beam and outputs that the intrinsic source may be as large as $0.55 \times 00^{\prime \prime} 09$.

The white contours in Figure 1 show the $1.2 \mathrm{~mm}$ continuum emission as measured from the 3 sub-bands without the $[\mathrm{C} \mathrm{II}]$ line. In contrast to the strong and clear $[\mathrm{C}$ II] emission the continuum in this source is very weak. 
There is a marginal continuum detection at the $2 \sigma$ level that is only considered as plausible true emission because its centroid co-incides to within 0.3 of the [C II] line centroid. There are several other peaks of this magnitude within the $6^{\prime \prime} \times 6^{\prime \prime}$ image of Figure 1 that may be due solely to noise. We note that there is an emerging trend in ALMA observations of non-ULIRG highredshift galaxies that the [C II] line emission is detected at much higher significance than the continuum, despite the large instantaneous bandwidth of $7.5 \mathrm{GHz}$ (Riechers et al. 2014, Willott et al. 2013a, 2015, Capak et al. 2015). We discuss the ratio of line to continuum emission further in Section 4

The NIR image in Figure 1 shows the location of the rest-frame UV continuum in CLM 1. The Ly $\alpha$ contribution to this flux is negligible. The galaxy is indistinguishable from a point source at the $0 . \prime 8$ resolution of the NIR data. The centroids of the $[\mathrm{C} I \mathrm{II}$ and rest-frame UV co-incide to within $0{ }^{\prime \prime} 1$, suggesting they trace the same star forming regions of the galaxy, at this resolution.

Visible to the east of CLM 1 is a lower redshift elliptical galaxy whose centroid is only $3^{\prime \prime}$ from CLM 1 . The presence of this galaxy combined with the high UV luminosity of CLM 1 raises the possibility that the high luminosity is in part due to magnification by gravitational lensing. To determine the potential lensing magnification we have analyzed the properties of this elliptical galaxy. We fit galaxy models to 12 band photometry from the CFHT Legacy Survey, VIDEO and AllWISE using the FAST code (Kriek et al. 2009). The spectral energy distribution (SED) is well fit by an old stellar population at $z=0.54$ with a stellar mass of $5 \times 10^{10}\left(M_{\odot}\right)$. Using the observed correlation between velocity dispersion and stellar mass (Wake et al. 2012) at $z=0.1$ and with negligible evolution from $z=0.5$ to $z=0.1$ (Shu et al. 2012) this gives a velocity dispersion of $126 \mathrm{~km} \mathrm{~s}^{-1}$. Assuming an isothermal sphere potential this lensing configuration provides a magnification factor of only 1.13. Given this relatively low magnification we do not make any lensing corrections to physical values in this paper.

Figure 2 plots the [C II] spectrum of CLM 1 and the Ly $\alpha$ spectrum for comparison. Interpretation of the velocity difference between $[\mathrm{C} \mathrm{II}]$ and $\mathrm{Ly} \alpha$ is deferred to Section 5. The [C II] spectrum is well fit by a single Gaussian plus a very low level, flat continuum. The fit continuum level is consistent with the low $\mathrm{S} / \mathrm{N}$ continuum detection from the other 3 sub-bands described above. There is marginal excess emission in the red wing. From the Gaussian peak frequency we determine the systemic redshift of the galaxy as $z=6.1657 \pm 0.0003$. The Gaussian FWHM is $162 \pm 23 \mathrm{~km} \mathrm{~s}^{-1}$. Table 1 contains further measurements made from the ALMA data.

With this velocity and the upper limit on the source size $(<0.55$ or $3.2 \mathrm{kpc})$ we can calculate an upper limit to the dynamical mass. Following the procedure of Wang et al. (2013) we derive $M_{\text {dyn }}<6.9 \times 10^{9} / \sin ^{2} i M_{\odot}$ where $i$ is the unknown inclination angle.

Using photometry from the rest-frame UV, optical and far-IR we fit galaxy spectral models to understand the evolutionary state of CLM 1. A synthetic ALMA continuum filter is generated using the wavelengths covered by the three line-free sub-bands. We use the Python implementation of the CIGALE package (Roehlly et al. 2012) to fit the SED. This code determines the ultraviolet-

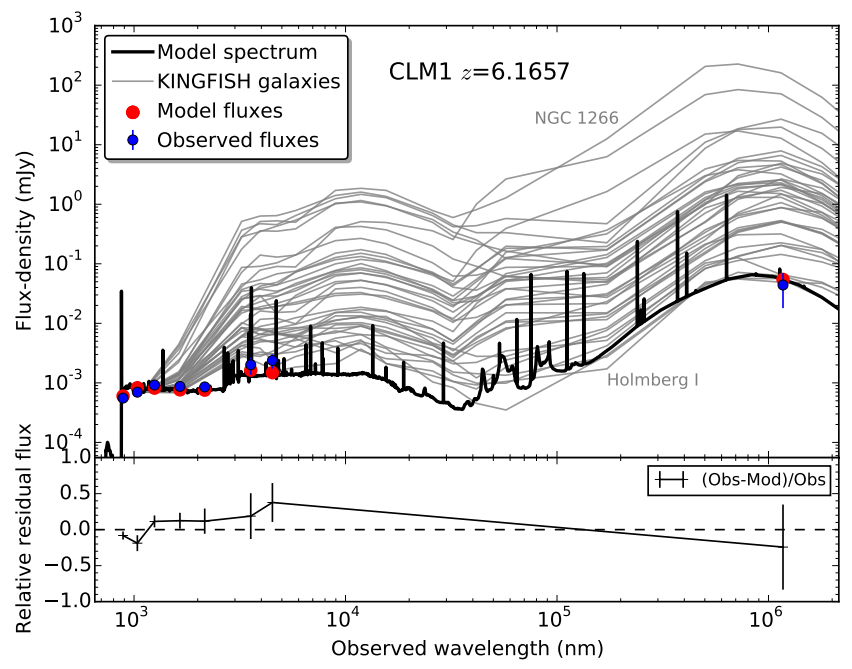

FIG. 3.- Observed-frame optical to far-IR SED of CLM 1 (blue circles). The best-fit model from CIGALE is shown as a black curve and the model fluxes through the observed filters as red circles. The grey curves are 39 nearby galaxy SEDs with UV to far-IR photometry from the KINGFISH survey (Dale et al. 2012) ranging from dusty infra-red galaxies to dwarf irregulars with very low thermal dust emission. The lower panel shows the residuals from the best-fit model. CLM 1 has a SED most similar to the least dusty nearby dwarf irregulars.

TABLE 1

Millimeter DATA FOR THE $z>6$ LBGs

\begin{tabular}{lll}
\hline & CLM 1 & WMH 5 \\
\hline$z_{\mathrm{Ly} \alpha}$ & $6.176 \pm 0.002$ & $6.076 \pm 0.001$ \\
$z_{[\mathrm{CII}]}$ & $6.1657 \pm 0.0003$ & $6.0695 \pm 0.0003^{\mathrm{a}}$ \\
& & $6.0639 \pm 0.0003 \mathrm{~b}$ \\
$\mathrm{FWHM}_{[\mathrm{CII}]}$ & $162 \pm 23 \mathrm{~km} \mathrm{~s}^{-1}$ & $251 \pm 15 \mathrm{~km} \mathrm{~s}^{-1} \mathrm{a}$ \\
& & $68 \pm 13 \mathrm{~km} \mathrm{~s}^{-1 \mathrm{~b}}$ \\
$I_{[\mathrm{CII}]}\left(\mathrm{Jy} \mathrm{km} \mathrm{s}^{-1}\right)$ & $0.234 \pm 0.031$ & $0.659 \pm 0.071$ \\
$L_{[\mathrm{CII}]}\left(L_{\odot}\right)$ & $(2.40 \pm 0.32) \times 10^{8}$ & $(6.60 \pm 0.72) \times 10^{8}$ \\
$f_{1.2 \mathrm{~mm}}(\mu \mathrm{Jy})$ & $44 \pm 26$ & $218 \pm 41$ \\
$L_{\mathrm{FIR}}\left(L_{\odot}\right)$ & $(2.5 \pm 1.5) \times 10^{10}$ & $(1.25 \pm 0.24) \times 10^{11}$ \\
$L_{[\mathrm{CII}]} / L_{\mathrm{FIR}}$ & $(9.5 \pm 5.7) \times 10^{-3}$ & $(5.3 \pm 1.2) \times 10^{-3}$ \\
$\mathrm{SFR}_{[\mathrm{CII}]}\left(M_{\odot} \mathrm{yr}^{-1}\right)$ & $24 \pm 3$ & $66 \pm 7$ \\
$\operatorname{SFR}_{\mathrm{FIR}}\left(M_{\odot \mathrm{yr}^{-1}}\right)$ & $7 \pm 4$ & $33 \pm 6$ \\
$\mathrm{SFR}_{\mathrm{SED}}\left(M_{\odot \mathrm{yr}^{-1}}\right)$ & $37 \pm 4$ & $43 \pm 5$ \\
\hline
\end{tabular}

Notes.-

a The redshift and FWHM of component 'A' only.

b The redshift and FWHM of component 'B' only.

Uncertainties in $L_{\text {FIR }}$ and SFR only include measurement uncertainties, not the uncertainties in extrapolating from a monochromatic to integrated luminosity, luminosity-SFR calibrations, or uncertainty in star formation history.

optical attenuation and its re-emission in the infrared. Stellar light is modeled with a single Bruzual \& Charlot (2003) stellar population with constant star formation rate, Chabrier initial mass function and metallicity $=0.008$. Dust attenuation followed the Calzetti et al. (2000) dependence on wavelength with re-emission in the IR via the SED parameterization of Dale et al. (2014).

The highest likelihood parameters are a stellar mass of $1.3 \times 10^{10} M_{\odot}, \mathrm{SFR}=37 M_{\odot} \mathrm{yr}^{-1}$ and minimal dust attenuation of $\mathrm{E}(\mathrm{B}-\mathrm{V})=0.01$. This very low amount of dust attenuation is constrained by the blue rest-frame UV slope of $\beta=-2.0$ (where $f_{\lambda} \propto \lambda^{\beta}$ ) and in an IR remission scenario consistent with the low FIR luminosity from the marginal $1.2 \mathrm{~mm}$ continuum detection. We 
note that an increasing star formation rate and/or higher fraction of the IRAC flux contributed by nebular emission lines could substantially decrease the stellar mass, bringing it more into line with the dynamical mass upper limit. It is also possible that the [C II]-emitting gas is not tracing the full dynamical mass of the system.

In Figure 3 we show the observed photometry of CLM 1 along with the best-fit model photometry, the model spectrum and, in the lower panel, the residuals from the fit. With almost as many free parameters as data points, a good fit has been found. The observed IRAC fluxes are slightly higher than the model suggesting possibly stronger nebular lines than modelled, although there are large uncertainties in the IRAC fluxes. The gray curves in Figure 3 show redshifted SEDs for nearby galaxies from the KINGFISH survey, normalized at restframe $150 \mathrm{~nm}$. These galaxies have been observed with GALEX, Spitzer Space Telescope, Herschel Space Observatory and in the optical and near-IR (Dale et al. 2007. 2012). The galaxies range in total IR luminosity from $10^{7}$ to $10^{11} L_{\odot}$. CLM 1 has a SED most similar to the $\sim 10^{7} L_{\odot}$ galaxies, despite having an IR luminosity of $>10^{10} L_{\odot}$, in essence it is a scaled up (by $10^{3}$ ) version of the low metallicity dwarf irregulars.

It has recently been shown that the [C II] luminosity is an effective tracer of the star formation rate in low redshift starbursts (De Looze et al. 2014, Sargsyan et al. 2014). In addition to the far-IR luminosity and SED (largely constrained by UV, rather than FIR, fluxes) this gives three independent measures of the SFR in this $z>$ 6 galaxy. For the [C II] luminosity we use the relation $\operatorname{SFR}\left(M_{\odot} \mathrm{yr}^{-1}\right)=1.0 \times 10^{-7} L_{[\mathrm{CII}]}\left(L_{\odot}\right)($ Sargsyan et al. 2014).

We calculate the far-IR luminosity $L_{\mathrm{FIR}}$ (integrated over rest-frame 42.5 to $122.5 \mu \mathrm{m}$ ) from the observed $1.2 \mathrm{~mm}$ continuum assuming a greybody spectrum with dust temperature, $T_{\mathrm{d}}=30 \mathrm{~K}$ and emissivity index, $\beta=$ 1.6. We use $T_{\mathrm{d}}=30 \mathrm{~K}$ because that is the dust temperature for similar FIR-luminosity galaxies at low-redshift (Symeonidis et al. 2013). We note that a higher dust temperature of $T_{\mathrm{d}}=45 \mathrm{~K}$ would increase the $L_{\mathrm{FIR}}$ value by a factor of about three. To convert from $L_{\text {FIR }}$ to SFR we use the relation $\operatorname{SFR}\left(M_{\odot} \mathrm{yr}^{-1}\right)=1.5 \times 10^{-10} L_{\mathrm{FIR}}\left(L_{\odot}\right)$ appropriate for a Chabrier IMF (Carilli \& Walter 2013).

At these redshifts the Cosmic Microwave Background (CMB) has a temperature of $19 \mathrm{~K}$ and can potentially bias measurements of dust continuum luminosity. There are two competing effects: (i) a high CMB background against which the continuum is measured, and (ii) an increase in the dust temperature due to heating by the CMB. Since we only have one continuum point and no constraints on dust temperature, we cannot make an accurate correction for these effects, but note that according to the analysis of da Cunha et al. (2013) the two effects are of comparable size and opposite sign for likely dust temperatures at this redshift, so we make no correction.

All three SFR estimates are listed in Table 1. For CLM 1 we find that $\mathrm{SFR}_{\mathrm{SED}}$ and $\mathrm{SFR}_{[\mathrm{CII}]}$ are comparable, but $\mathrm{SFR}_{\text {FIR }}$ is lower than $\mathrm{SFR}_{[\mathrm{CII}]}$ (see Section 4) and lower than $\mathrm{SFR}_{\mathrm{SED}}$, as expected from the low dust contribution to the SED in Figure 3. Previous studies have shown that $\mathrm{SFR}_{\mathrm{FIR}}$ may be unreliable as a tracer of the total
SFR in very low dust and/or metallicity galaxies (Ouchi et al. 2013; Fisher et al. 2014, Ota et al. 2014). An alternative is that a higher dust temperature of $T_{\mathrm{d}}=45 \mathrm{~K}$ would raise $\mathrm{SFR}_{\mathrm{FIR}}$ to a level comparable with $\mathrm{SFR}_{\mathrm{SED}}$ and $\mathrm{SFR}_{[\mathrm{CII}]}$. Future observations at shorter wavelength are critical to constrain the full IR SED of high- $z$ galaxies and constrain the dust temperature.

\section{2. $W M H 5$}

The ALMA and NIR images for WMH 5 are shown in Figure 4. There is a much more firm $1.2 \mathrm{~mm}$ continuum detection in this source at significance $6 \sigma$. The beam is more circular for WMH 5 than for CLM 1 and both the [C II] and $1.2 \mathrm{~mm}$ continuum are clearly spatially extended at $\mathrm{PA} \approx 80$. The $[\mathrm{C}$ II $]$ emission centroid shows a significant offset from the NIR emission of 0 ". 4 . The NIR emission is consistent with a point source. The Ly $\alpha$ emission is also spatially unresolved along the slit direction.

Figure 5 shows the [C II] spectrum of WMH 5. The line can be split into two Gaussian components. We label the component with higher flux and larger linewidth $\left(\mathrm{FWHM}=251 \mathrm{kms}^{-1}\right.$ ) as ' $\mathrm{A}$ ' and the other component as 'B' $\left(\mathrm{FWHM}=68 \mathrm{~km} \mathrm{~s}^{-1}\right)$. Assuming the linewidths trace mass gravitationally we identify the systemic redshift of the main galaxy WMH 5 with the velocity of 'A'. To determine the nature of this multiple velocity component system we show in Figure 6 a positionvelocity diagram along the major axis of [CII] emission. The separation of the two components in velocity is clear, but we also find a spatial offset of 0.73 (2 kpc) between the peaks of the two components. In Figure 4 we mark the centroids of components 'A' and 'B' separately to highlight how those positions compare with each other and the NIR emission. Both [C II] components are marginally resolved with intrinsic major axis sizes of 0 '. $48 \pm 0$ ". 1 and 0 .' $4 \pm 0^{\prime \prime} .1$ for ' $\mathrm{A}$ ' and ' $\mathrm{B}$ ', respectively. We determine dynamical masses from the $[\mathrm{C} \mathrm{II}]$ sizes and FWHM of $M_{\text {dyn }}=1.7 \times 10^{10} / \sin ^{2} i M_{\odot}$ for $\mathrm{A}$ and $M_{\mathrm{dyn}}=1.0 \times 10^{9} / \sin ^{2} i M_{\odot}$ for B.

We carry out SED-fitting using CIGALE for the entire WMH 5 system. The observed and modelled SEDs are shown in Figure 7. As for CLM 1, the photometry is well fit by a single stellar population whose attenuated UV emission is re-radiated in the FIR. The highest likelihood parameters are a stellar mass of $2.3 \times 10^{10} M_{\odot}, \mathrm{SFR}=$ $43 M_{\odot} \mathrm{yr}^{-1}$ and dust attenuation of $\mathrm{E}(\mathrm{B}-\mathrm{V})=0.05$. The higher dust attenuation in WMH 5 is constrained by the redder UV spectral slope and consistent with the higher $L_{\mathrm{FIR}}$. However, in this system we know that there is a spatial offset between the UV and FIR, so such a simple scenario of attenuation and re-emission is not physically plausible.

From this analysis the system appears to be an ongoing merger of two galaxies. ' $\mathrm{B}$ ' is apparently lower mass but is spatially coincident with the NIR emission. This is similar to some lower redshift ULIRG mergers Chapman et al. 2004) with an optically obscured component that dominates the FIR emission and a lower $L_{\text {FIR }}$ component less obscured by dust. Similar cases of most of the [C II] associated with optically-faint components have been reported at $z>5$ by Capak et al. (2015); Maiolino et al. (2015), however in our case com- 

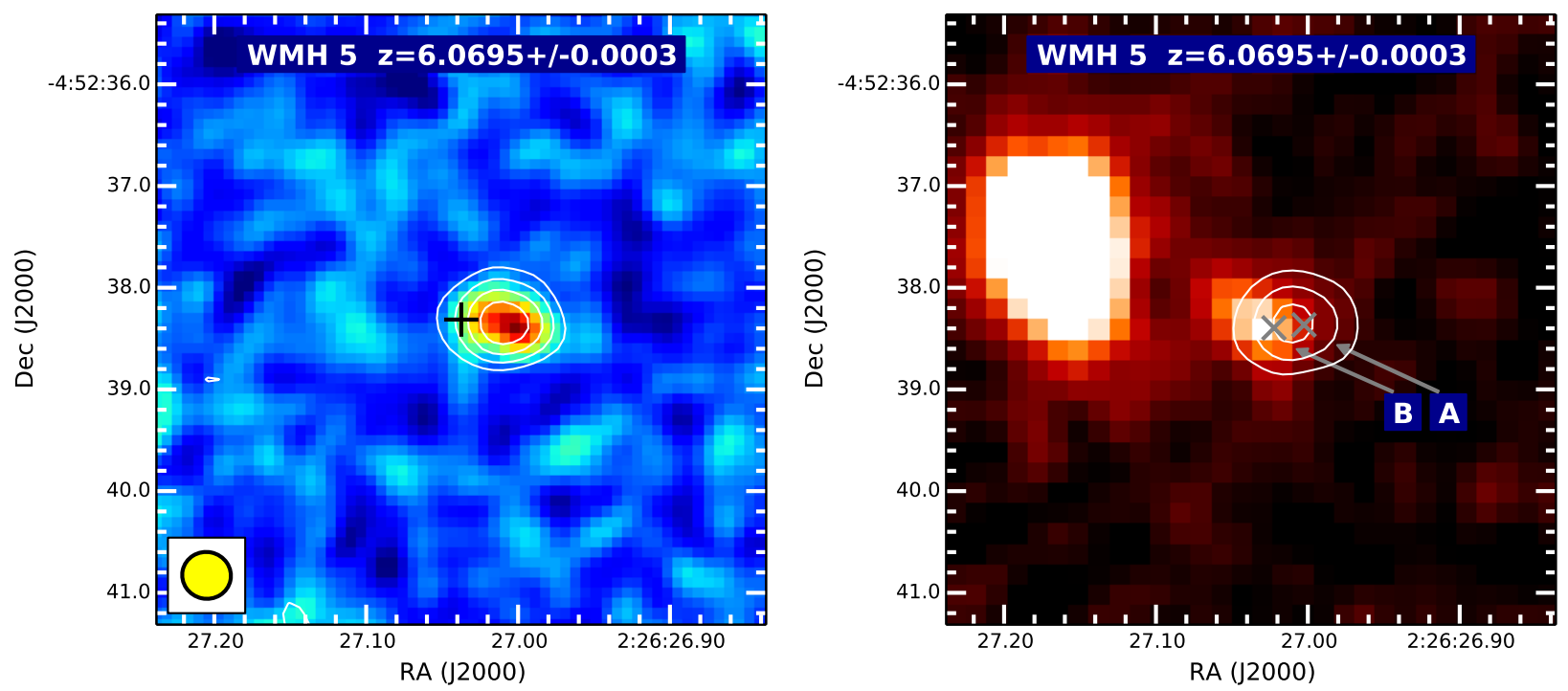

FIG. 4.- Left: The background image is ALMA integrated [C II] line map of WMH 5. White contours show the clear $1.2 \mathrm{~mm}$ dust continuum detection (contour levels 2,3,4,5 $\sigma$ beam $^{-1}$ ). The NIR centroid (black plus symbol) is significantly offset to the east.

Right: The background is the $z Y J$ NIR image. Contours show the ALMA [C II] emission from the left panel at contour levels $3,6,9 \sigma$ beam ${ }^{-1}$. Grey crosses labeled 'A' and 'B' correspond to the centroids of the two [C II] velocity components. The rest-frame UV continuum is consistent with 'B', the component with lower [C II] luminosity and velocity width. There is a foreground galaxy further east of the system.
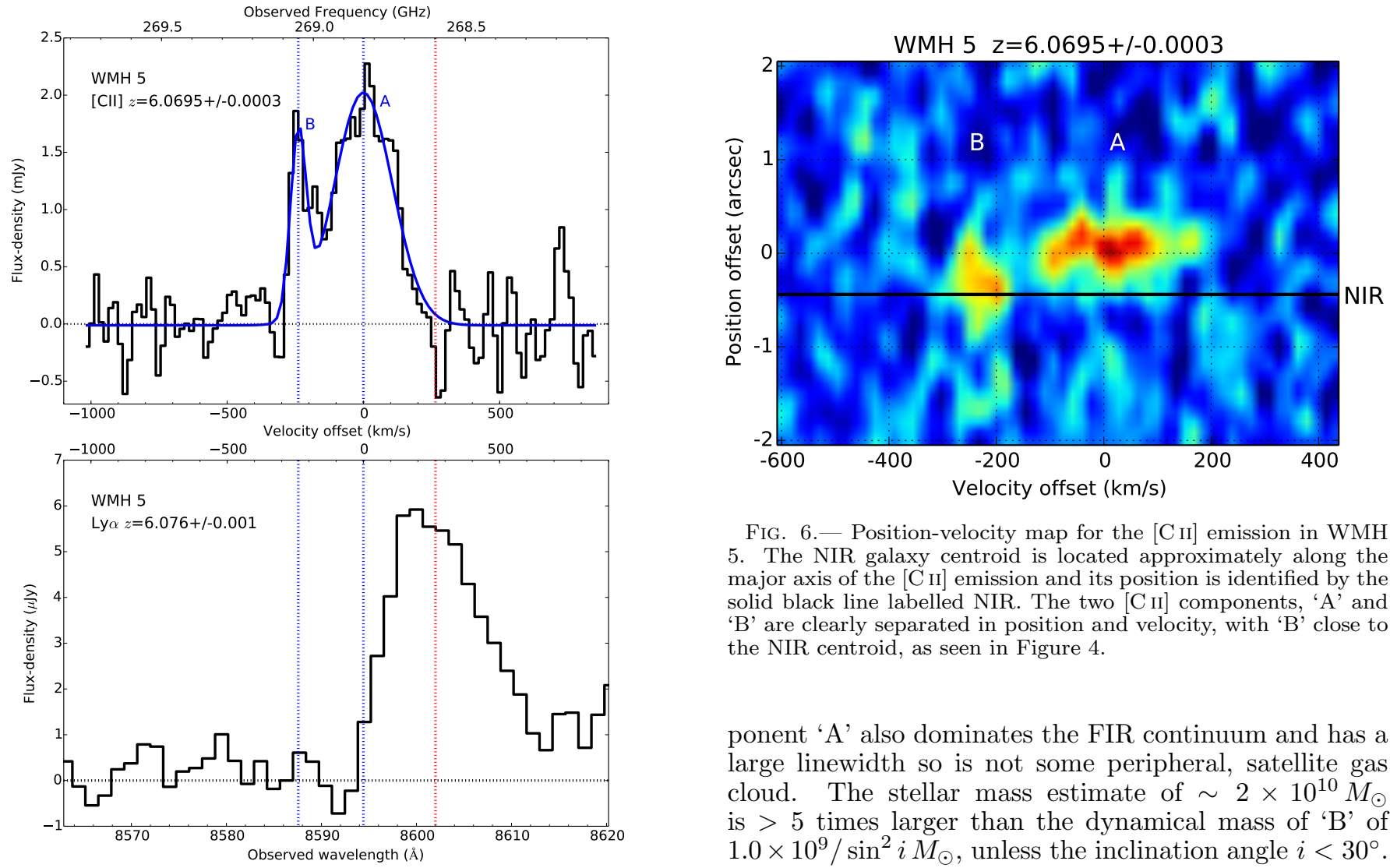

Fig. 5.- ALMA [C II] (upper) and Gemini GMOS Ly $\alpha$ (lower) spectra of WMH 5 (see Figure 2 for details). The [C II] data have been continuum-subtracted in the image plane. For this galaxy there are two [CII] velocity components ('A' and 'B'), that are each well fit by a Gaussian. The systemic redshift is taken to be the Gaussian peak of ' $\mathrm{A}$ ' as this is the most luminous and highest width component. The Ly $\alpha$ spectrum is asymmetric and the fluxweighted centroid offset by $+265 \pm 52 \mathrm{~km} \mathrm{~s}^{-1}$ from 'A' and $+504 \pm$ $52 \mathrm{~km} \mathrm{~s}^{-1}$ from 'B'.

FIG. 6.- Position-velocity map for the [C II] emission in WMH 5. The NIR galaxy centroid is located approximately along the major axis of the $[\mathrm{C} \mathrm{II}]$ emission and its position is identified by the solid black line labelled NIR. The two [C II] components, ' $\mathrm{A}$ ' and ' $\mathrm{B}$ ' are clearly separated in position and velocity, with ' $\mathrm{B}$ ' close to the NIR centroid, as seen in Figure 4

ponent ' $\mathrm{A}$ ' also dominates the FIR continuum and has a large linewidth so is not some peripheral, satellite gas cloud. The stellar mass estimate of $\sim 2 \times 10^{10} M_{\odot}$ is $>5$ times larger than the dynamical mass of ' $\mathrm{B}$ ' of $1.0 \times 10^{9} / \sin ^{2} i M_{\odot}$, unless the inclination angle $i<30^{\circ}$. Either ' $\mathrm{B}$ ' is a disk viewed extremely face-on or it is not responsible for all the stellar mass.

For WMH 5 the SFR estimates from the far-IR, [C II] and SED-fitting in Table 1 are all within a factor of two. This is rather surprising given the complex nature of the system. The millimeter and UV SFR have similar values, but their spatial displacement indicates that they are not probing the same star-forming regions, so the true SFR should be the sum of both components. 


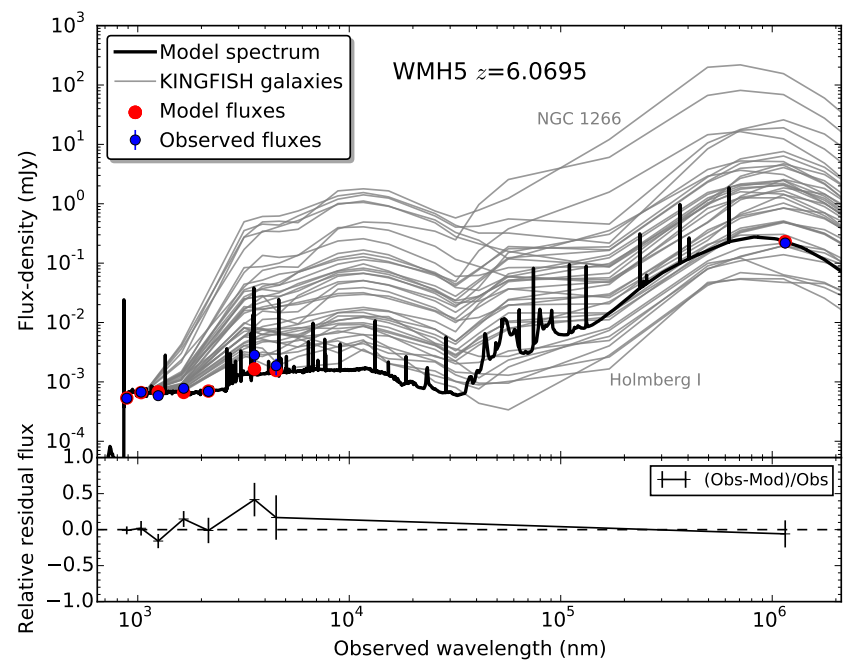

FIG. 7.- Observed-frame optical to far-IR SED of WMH 5 (blue circles), best-fit CIGALE model and SEDs of nearby KINGFISH survey galaxies (see Figure 3 for details). WMH 5 has a SED most similar to nearby dwarf irregulars.

\section{THE [C II] - FAR-IR LUMINOSITY RELATION}

For both $[\mathrm{CII}]$ and FIR luminosities to act as reliable star formation rate indicators requires that the ratio of the two behave in a predictable manner without too high a scatter. At low-redshift most galaxies have ratios in the range $10^{-3}$ to $10^{-2}$ with a fairly linear relationship between the two luminosities (De Looze et al. 2014 Sargsyan et al. 2014). The exception to this is in the ULIRG regime where a deficit of [C II] luminosity is usually observed, thought to be related to extreme densities and temperatures in circumnuclear starburst regions (Farrah et al. 2013; Magdis et al. 2014; González-Alfonso et al. 2015). Figure 8 plots the low redshift data with small black circles.

At higher redshift $(z>4)$, the first [C II] measurements were made in very high $L_{\mathrm{FIR}}$ sources and showed low $[\mathrm{CII}] / \mathrm{FIR}$ ratios comparable with nearby ULIRGs (Maiolino et al. 2005, Iono et al. 2006). Subsequent work at redshifts between 1 and 7 revealed a wide range of ratios from $10^{-4}$ to $10^{-1}$. At the low end there are highluminosity $z>5.7$ quasars (Wang et al. 2013) and hyperluminous infrared galaxies (HyLIRGS; $L_{\text {FIR }}>10^{13} L_{\odot}$ ) (Riechers et al. 2013, 2014). At the opposite end of the range, high $[\mathrm{C}$ II $] / \mathrm{FIR}$ ratios up to $10^{-1}$ have been found in some massive $1>z>2$ ULIRGs lying on the mainsequence of star-forming galaxies (Stacey et al. 2010 Brisbin et al. 2015). In these galaxies, the star-formation is more spatially extended than in nearby ULIRGs leading to higher [C II] luminosities.

In Figure 8 we also include recent ALMA observations of $L_{\mathrm{FIR}}<10^{12} L_{\odot}$ objects comprising $z>6 \mathrm{AGN}$ (Willott et al. 2013a, 2015) and $z>5$ galaxies (Capak et al. 2015; Maiolino et al. 2015) plus CLM 1 and WMH 5. Our data are the first non-limit data at $z>6$ in $L_{\mathrm{FIR}}<10^{12} L_{\odot}$ galaxies without AGN. For $z>5$ galaxies the $[\mathrm{C} \mathrm{II}] /$ FIR ratios display a broad range at somewhat higher values than at low-redshift. Ratios of $\sim 10^{-2}$ suggest extended star-formation with low metallicity and an intense radiation field.

The similarity of high-redshift galaxies hosting quasars

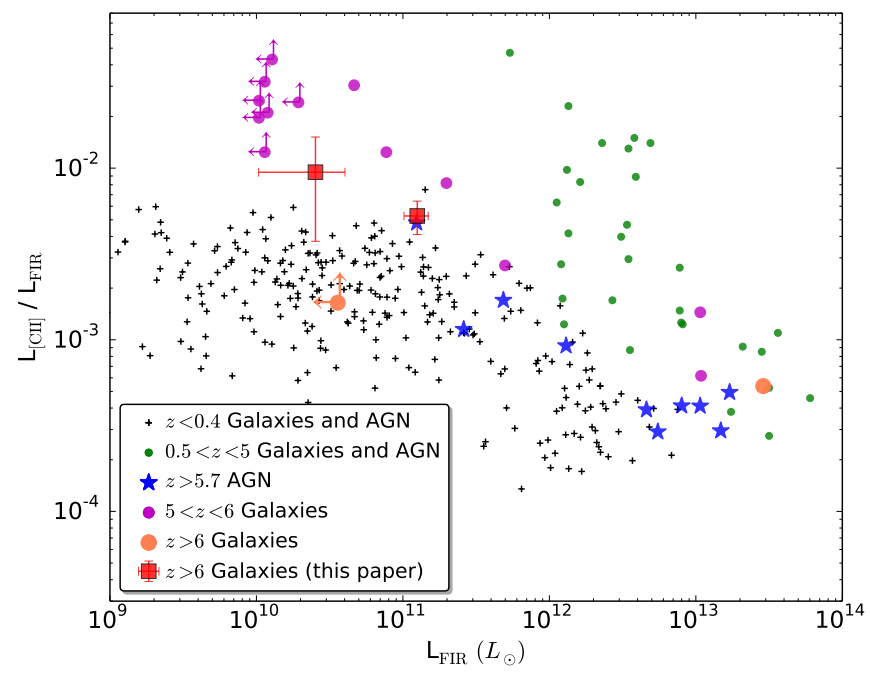

FIG. 8.- Ratio of [C II] to far-IR ( 42.5 to $122.5 \mu \mathrm{m}$ ) luminosity versus far-IR luminosity. The two $z>6$ LBGs from this paper are plotted as red squares with error bars. Other galaxies detected in the [C II] transition at $z>6$ plotted as orange circles are (leftto-right) BDF3299 Clump A at $z=7.107$ (Maiolino et al. 2015) and HFLS 3 at $z=6.337$ (Riechers et al. 2013). Galaxies at $5<z<6$ (magenta circles) are trom Rawle et al. (2014); Riechers et al. (2014); Capak et al. (2015) where the Capak et al. total IR Iuminosities have been divided by a factor 1.75 to give $L_{\mathrm{FIR}}$. AGNhosting galaxies at $z>5.7$ are shown with blue stars (Maiolino et al. 2005, Venemans et al. 2012, Wang et al. 2013, Willott et al. 2013a 2015). Galaxies (including some AGN) at $0.5<z<5$ (green circles) are from Brisbin et al. (2015) and the compilation of De Looze et al. (2014) and at $z<0.4$ (black plus symbol) from the compilation of Graciá-Carpio et al. (2011 and in prep.). The $z>6$ LBGs CLM 1 and WMH 5 have ratios comparable to $z>5$ galaxies of similar $L_{\mathrm{FIR}}$, somewhat higher than at $z<0.4$.

and without quasars gives us confidence that the star formation properties are only weakly affected by black hole accretion onto moderate mass black holes of $M_{\mathrm{BH}} \sim$ $10^{8} M_{\odot}$, compared to the very low $[\mathrm{CII}] / \mathrm{FIR}$ values found in the more luminous (in both accretion and FIR) $z>5 M_{\mathrm{BH}} \sim 10^{9} M_{\odot}$ quasars of Wang et al. (2013). We note that the $L_{\mathrm{FIR}}<10^{12} L_{\odot} z>6 \mathrm{AGN}$ have observed ratios $f_{1.2 \mathrm{~mm}} / I_{[\mathrm{CII}]}$ similar to CLM 1 and WMH 5 , their lower ratios in this figure being due to the factor of 4 higher $L_{\text {FIR }}$ calculated using a dust temperature of $T_{d}=47 \mathrm{~K}$, compared to $T_{d}=30 \mathrm{~K}$ for the galaxies.

\section{Ly $\alpha$ EMISSION LINE VELOCITY SHIFTS AND INTERPRETATION OF RAPID EVOLUTION IN $Z \approx 7$ Ly $\alpha$ GALAXIES}

Atomic or molecular gas in star forming regions spread throughout galaxies is ideal for measuring the systemic redshift of the galaxy. At high redshift such measurements have been used to determine the ionized bubble sizes surrounding luminous quasars (Carilli et al. 2010). In this Section we use this information to determine the velocity shifts of the observed Ly $\alpha$ emission from CLM 1 and WMH 5. Ly $\alpha$ at $z>6$ is the most important probe of the fraction of neutral hydrogen in the IGM and the process of cosmic reionization.

In Figures 2 and 5 we plotted the [C II] and Ly $\alpha$ spectra on the same velocity scale after correcting the datasets to the local standard of rest. For both galaxies the Ly $\alpha$ line is asymmetric with a broader red wing than blue and the line center is shifted to the red from the systemic 
redshift defined by the $[\mathrm{C} I \mathrm{II}$ observations. This highly asymmetric line shape is characteristic of $\mathrm{Ly} \alpha$ at highredshift (Shimasaku et al. 2006) due to neutral hydrogen absorption of the blue wing. For CLM 1 the measured offset is $+430 \pm 69 \mathrm{~km} \mathrm{~s}^{-1}$ and the Ly $\alpha$ flux drops to zero just before the $[\mathrm{C} \mathrm{II}]$ line center. For WMH 5 there is uncertainty in the systemic redshift due to the two components. If ' $\mathrm{A}$ ', the component with larger [C II] linewidth and therefore likely higher mass, is to be identified as the Ly $\alpha$ systemic redshift then the offset is $+265 \pm 52 \mathrm{~km} \mathrm{~s}^{-1}$. However, the astrometry suggests that ' $\mathrm{B}$ ' is the origin of the rest-frame UV and therefore $\operatorname{Ly} \alpha$ emission and in this case the shift is $+504 \pm 52 \mathrm{~km} \mathrm{~s}^{-1}$. For the remainder of this section we consider both cases as possible.

The emergence of Ly $\alpha$ from galaxies is a complicated process that involves resonant scattering off neutral hydrogen and absorption by dust. The observed Ly $\alpha$ profiles depend upon factors such as geometry, gas covering factor, dust and outflow velocity. Irrespective of the ionization state of the IGM, galaxies tend to show Ly $\alpha$ profiles offset to the red due to resonant scattering and selective absorption within galactic-scale outflows (see review in Dijkstra 2014). At $z>6$ where the neutral fraction of the IGM becomes significant, it is expected that IGM absorption of Ly $\alpha$ decreases the overall Ly $\alpha$ flux and shifts the line center further to the red (Laursen et al. 2011). The very sharp decrease in Ly $\alpha$ line strength of LBGs between $z=6$ and $z=7$ has been interpreted as evidence for a rapid change in IGM neutral fraction at this epoch, in tension with reionization models that predict a smoother change in neutral fraction over this short cosmic time (Pentericci et al. 2011, Schenker et al. 2012 Treu et al. 2013).

The first measurement of Ly $\alpha$ velocity shifts at redshifts relevant to the reionization epoch have recently been made by Stark et al. (2014a). These authors used the UV nebular C III $\lambda 1909$ doublet, which is a relatively strong line in young, low-mass star-forming galaxies (Stark et al. 2014b), to determine the systemic redshifts of two strong Ly $\alpha$ emitters. The gravitationallylensed $z=6.027$ galaxy A383-5.2 has a high Ly $\alpha$ equivalent width with strong asymmetry. Stark et al. (2014a) quote the $\operatorname{Ly} \alpha$ peak velocity offset as $120 \mathrm{~km} \mathrm{~s}^{-1}$. For comparison with our velocity offsets and previous measurements at lower redshift we determine from their Figure 5 an approximate centroid-based velocity offset for this galaxy of $150 \pm 30 \mathrm{~km} \mathrm{~s}^{-1}$. The $z=7.213$ galaxy GN108036 has a more moderate Ly $\alpha$ equivalent width and a much lower significance detection of $\mathrm{CIII}$ ] was obtained by Stark et al. (2014a) with a very low and uncertain velocity offset of $-37 \pm 113 \mathrm{~km} \mathrm{~s}^{-1}$.

Stark et al. (2014a) noted that these offsets are small compared to those of LBGs at lower redshift and this may be due to different physical conditions at high redshift. In particular there is known to be a negative correlation between velocity shift and Ly $\alpha$ equivalent width (Shibuya et al. 2014, Erb et al. 2014) and Ly $\alpha$ strengths increase with redshift up to $z=6$ (Stark et al. 2011). Since Ly $\alpha$ more easily escapes from galaxies via the wings than the line center, a decrease in velocity shift at highredshift could be partially responsible for the sudden decrease in Ly $\alpha$ emitters at this epoch. Choudhury et al. (2014) showed that a negative evolution of velocity shift with redshift can match the observed $\operatorname{Ly} \alpha$ equivalent

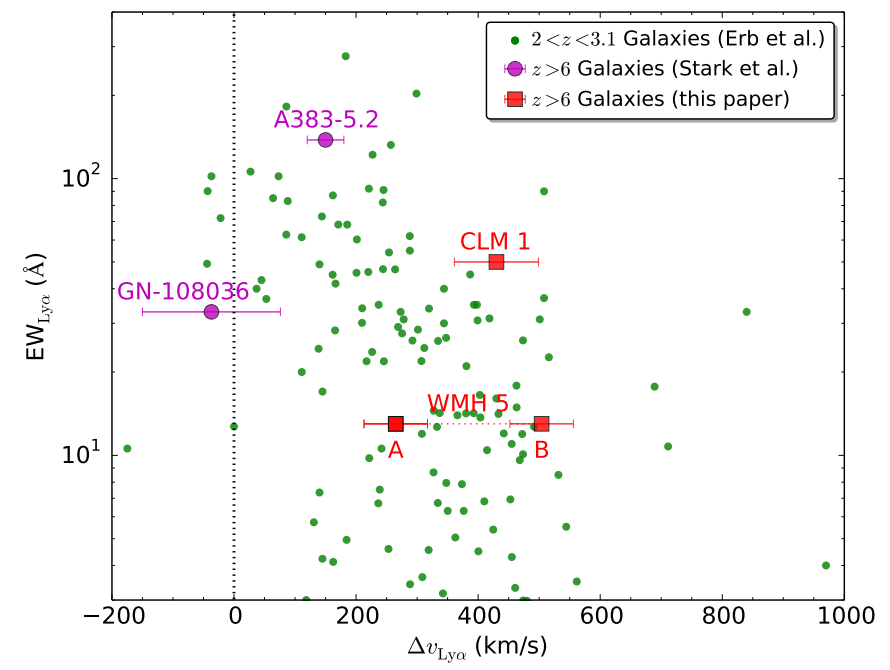

FIG. 9. - Rest-frame equivalent width of the Ly $\alpha$ emission line versus the velocity offset between Ly $\alpha$ and the systemic redshift. The two $z>6$ LBGs from this paper are plotted as red squares with error bars. For WMH 5 the two [CII] components, 'A' and 'B', provide two estimates of the velocity offset connected by a dotted line. Magenta circles show the only other two galaxies at such high redshifts with measured Ly $\alpha$ velocity offsets (Stark et al. 2014a) based on the offset to the C III] $\lambda 1909$ UV emission line. A comparison sample of galaxies at intermediate redshift from Erb et al. (2014 also Strom et al. in prep.) is plotted as green circles. If the systemic redshift of the WMH 5 Ly $\alpha$ emission is given by ' $\mathrm{B}$ ', then the two $z>6$ galaxies from this paper lie at the large velocity shift end of the distribution defined by the intermediate redshift sample.

width distribution evolution to smooth reionization history models (see also Bolton \& Haehnelt 2013).

In Figure 9 we compare the Ly $\alpha$ velocity shifts measured for CLM 1 and WMH 5 to those of A383-5.2 and GN-108036. We choose to plot the shifts as a function of observed Ly $\alpha$ rest-frame equivalent width because of the correlation between velocity shift and Ly $\alpha$ equivalent width. In addition to these $z>6$ galaxies we show for comparison a sample selected at lower redshift $(2<z<3.1)$ as LAEs and LBGs (Erb et al. 2014, Strom et al. in prep.). For the lower redshift sample, spectroscopic equivalent widths are plotted when available, otherwise photometric equivalent widths are used. Several things are evident from looking at Figure 9 The shifts for CLM 1 and WMH 5 are larger than for A383-5.2 and GN-108036. None of the high-redshift galaxies have shifts at odds with those observed at $2<z<3.1$ when compared to galaxies with similar $\operatorname{Ly} \alpha$ equivalent width. If we adopt ' $\mathrm{B}$ ' as the systemic redshift for WMH 5, then both galaxies in this paper have $\mathrm{Ly} \alpha$ velocity shifts at the high end of the distribution, consistent with additional IGM absorption at higher redshift as predicted by the models of Laursen et al. (2011). Our analysis shows no observational evidence for velocity shifts that decrease as a function of redshift, at a given Ly $\alpha$ equivalent width. Obviously a much larger sample of observed high-redshift galaxies compared to realistic simulations incorporating radiative transfer would be required to use the $\operatorname{Ly} \alpha$ velocity shift distribution to constrain the details of reionization.

\section{CONCLUSIONS}


We have presented ALMA [C II] line and dust continuum detections of two UV-luminous LBGs at redshift $z>6$. These detections were made in relatively short integrations in Early Science operations providing hope that other galaxies in the reionization epoch with lower SFR will be detectable with the full ALMA array. Our results, in accord with other recent ALMA observations (Capak et al. 2015), confirm that, despite unexpectedly low $L_{\text {FIR, }}$ the increase in the $[\mathrm{C}$ II]/FIR ratio at highredshift leads to $[\mathrm{C}$ II] lines that are bright and very useful tracers of the ISM, as predicted by Walter \& Carilli (2008). The resolution of the merger of WMH 5 with a fairly compact ALMA array illustrates the power of [C II] line observations to understand the details of star formation and galaxy assembly at this epoch.

CLM 1 and WMH 5 were selected for study because their UV luminosity, stellar mass and redshift are comparable to the galaxy Himiko which was unexpectedly undetected by ALMA (Ouchi et al. 2013, Ota et al. 2014). The main difference between our target galaxies and Himiko (and most other high- $z$ non-detections) are our relatively low Ly $\alpha$ equivalent widths. Since the Ly $\alpha$ line strength decreases with increasing dust (and hence metallicity), this could explain the difference between these results. We note that the recent success with ALMA at $5<z<6$ is also for UV-luminous galaxies with relatively weak Ly $\alpha$ (Capak et al. 2015).

However, the UV slopes of our galaxies are fairly blue, indicating little dust absorption of the UV photons and we do observe a deficit of FIR photons compared to expectations, as shown by the SEDs that are similar to nearby dwarf irregulars, not with nearby galaxies with similar SFRs of tens of solar masses per year. Future observations at shorter wavelength are critical to constrain the full IR SED of high- $z$ galaxies and constrain the dust temperature. Are they very cool like low metal- licity dwarfs, despite the higher UV photon density and CMB temperature? Determining this is important as the full IR SED is required to derive more accurate values of $\mathrm{SFR}_{\text {FIR }}$ to determine whether this is a more or less accurate SFR indicator at high-z than $\mathrm{SFR}_{[\mathrm{CII}]}$ or $\mathrm{SFR}_{\mathrm{UV}}$.

We have for the first time used millimeter lines to determine Ly $\alpha$ line velocity offsets from systemic for galaxies in the reionization era. We find that the shifts in our two galaxies are comparable to the high end of the distribution at lower redshift, consistent with predictions from Ly $\alpha$ line asymmetry due to neutral hydrogen absorption (Laursen et al. 2011). More detailed studies in the future of spatially-resolved kinematics of [C II] and Ly $\alpha$ in such galaxies will allow stronger constraints to be placed on the escape of $\operatorname{Ly} \alpha$ emission from galaxies and its subsequent absorption by the IGM.

Thanks to staff at the North America ALMA Regional Center for processing the ALMA data, JeanGabriel Cuby for providing the optical spectrum of CLM 1, Dawn Erb for providing unpublished data on intermediate redshift Lyman Break Galaxies, and Peter Capak and Caitlin Casey for interesting discussions. This paper makes use of the following ALMA data: ADS/JAO.ALMA\#2013.1.00815.S. ALMA is a partnership of ESO (representing its member states), NSF (USA) and NINS (Japan), together with NRC (Canada) and NSC and ASIAA (Taiwan), in cooperation with the Republic of Chile. The Joint ALMA Observatory is operated by ESO, AUI/NRAO and NAOJ. The National Radio Astronomy Observatory is a facility of the National Science Foundation operated under cooperative agreement by Associated Universities, Inc.

Facility: ALMA.

\section{REFERENCES}

Bolton, J. S., \& Haehnelt, M. G. 2013, MNRAS, 429, 1695

Bouwens, R. J., Illingworth, G. D., Oesch, P. A., et al. 2014a, ApJ, 793,115

-. 2014b, ArXiv e-prints, arXiv:1403.4295

Brisbin, D., Ferkinhoff, C., Nikola, T., et al. 2015, ApJ, 799, 13

Bruzual, G., \& Charlot, S. 2003, MNRAS, 344, 1000

Calzetti, D., Armus, L., Bohlin, R. C., et al. 2000, ApJ, 533, 682

Capak, P. L., Carilli, C., Jones, G., et al. 2015, ArXiv e-prints, arXiv: 1503.07596

Carilli, C. L., \& Walter, F. 2013, ARA\&A, 51, 105

Carilli, C. L., Wang, R., Fan, X., et al. 2010, ApJ, 714, 834

Chapman, S. C., Smail, I., Windhorst, R., Muxlow, T., \& Ivison, R. J. 2004, ApJ, 611, 732

Choudhury, T. R., Puchwein, E., Haehnelt, M. G., \& Bolton, J. S. 2014, ArXiv e-prints, arXiv: 1412.4790

Cuby, J.-G., Le Fèvre, O., McCracken, H., et al. 2003, A\&A, 405, L19

Cutri, R. M., Wright, E. L., Conrow, T., et al. 2013, Explanatory Supplement to the AllWISE Data Release Products, Tech. rep., IPAC

da Cunha, E., Groves, B., Walter, F., et al. 2013, ApJ, 766, 13

Dale, D. A., Helou, G., Magdis, G. E., et al. 2014, ApJ, 784, 83

Dale, D. A., Gil de Paz, A., Gordon, K. D., et al. 2007, ApJ, 655, 863

Dale, D. A., Aniano, G., Engelbracht, C. W., et al. 2012, ApJ, 745, 95

De Looze, I., Cormier, D., Lebouteiller, V., et al. 2014, A\&A, 568, A62

Dijkstra, M. 2014, PASA, 31, 40

Erb, D. K., Steidel, C. C., Trainor, R. F., et al. 2014, ApJ, 795, 33
Farrah, D., Lebouteiller, V., Spoon, H. W. W., et al. 2013, ApJ, 776,38

Finlator, K., Oh, S. P., Özel, F., \& Davé, R. 2012, MNRAS, 427, 2464

Fisher, D. B., Bolatto, A. D., Herrera-Camus, R., et al. 2014, Nature, 505, 186

González-Alfonso, E., Fischer, J., Sturm, E., et al. 2015, ApJ, 800, 69

González-López, J., Riechers, D. A., Decarli, R., et al. 2014, ApJ, 784, 99

Graciá-Carpio, J., Sturm, E., Hailey-Dunsheath, S., et al. 2011, ApJ, 728, L7

Iono, D., Yun, M. S., Elvis, M., et al. 2006, ApJ, 645, L97

Jarvis, M. J., Bonfield, D. G., Bruce, V. A., et al. 2013, MNRAS, 428,1281

Kanekar, N., Wagg, J., Ram Chary, R., \& Carilli, C. L. 2013, ApJ, 771, L20

Kriek, M., van Dokkum, P. G., Labbé, I., et al. 2009, ApJ, 700, 221

Laursen, P., Sommer-Larsen, J., \& Razoumov, A. O. 2011, ApJ, 728,52

Magdis, G. E., Rigopoulou, D., Hopwood, R., et al. 2014, ApJ, 796, 63

Maiolino, R., Cox, P., Caselli, P., et al. 2005, A\&A, 440, L51

Maiolino, R., Carniani, S., Fontana, A., et al. 2015, ArXiv e-prints, arXiv: 1502.06634

Mancone, C. L., Gonzalez, A. H., Moustakas, L. A., \& Price, A. 2013, PASP, 125, 1514

Mauduit, J.-C., Lacy, M., Farrah, D., et al. 2012, PASP, 124, 714

Ono, Y., Ouchi, M., Curtis-Lake, E., et al. 2013, ApJ, 777, 155

Ota, K., Walter, F., Ohta, K., et al. 2014, ApJ, 792, 34 
Ouchi, M., Ellis, R., Ono, Y., et al. 2013, ApJ, 778, 102

Peng, C. Y., Ho, L. C., Impey, C. D., \& Rix, H.-W. 2010, AJ, 139, 2097

Pentericci, L., Fontana, A., Vanzella, E., et al. 2011, ApJ, 743, 132

Planck Collaboration, Ade, P. A. R., Aghanim, N., et al. 2015, ArXiv e-prints, arXiv:1502.01589

Rawle, T. D., Egami, E., Bussmann, R. S., et al. 2014, ApJ, 783, 59

Reddy, N., Dickinson, M., Elbaz, D., et al. 2012, ApJ, 744, 154

Riechers, D. A., Bradford, C. M., Clements, D. L., et al. 2013, Nature, 496, 329

Riechers, D. A., Carilli, C. L., Capak, P. L., et al. 2014, ApJ, 796, 84

Roehlly, Y., Burgarella, D., Buat, V., et al. 2012, in Astronomical Society of the Pacific Conference Series, Vol. 461, Astronomical Data Analysis Software and Systems XXI, ed. P. Ballester, D. Egret, \& N. P. F. Lorente, 569

Sargsyan, L., Samsonyan, A., Lebouteiller, V., et al. 2014, ApJ, 790,15

Schaerer, D., Boone, F., Zamojski, M., et al. 2015, A\&A, 574, A19

Schaerer, D., \& de Barros, S. 2010, A\&A, 515, A73

Schenker, M. A., Stark, D. P., Ellis, R. S., et al. 2012, ApJ, 744, 179

Shibuya, T., Ouchi, M., Nakajima, K., et al. 2014, ApJ, 788, 74

Shimasaku, K., Kashikawa, N., Doi, M., et al. 2006, PASJ, 58, 313

Shu, Y., Bolton, A. S., Schlegel, D. J., et al. 2012, AJ, 143, 90

Siana, B., Shapley, A. E., Kulas, K. R., et al. 2015, ArXiv e-prints, arXiv: 1502.06978
Smit, R., Bouwens, R. J., Labbé, I., et al. 2014, ApJ, 784, 58

Stacey, G. J., Hailey-Dunsheath, S., Ferkinhoff, C., et al. 2010, ApJ, 724, 957

Stark, D. P., Ellis, R. S., \& Ouchi, M. 2011, ApJ, 728, L2

Stark, D. P., Richard, J., Charlot, S., et al. 2014a, ArXiv e-prints, arXiv: 1408.3649

Stark, D. P., Richard, J., Siana, B., et al. 2014b, MNRAS, 445, 3200

Symeonidis, M., Vaccari, M., Berta, S., et al. 2013, MNRAS, 431, 2317

Treu, T., Schmidt, K. B., Trenti, M., Bradley, L. D., \& Stiavelli, M. 2013, ApJ, 775, L29

Venemans, B. P., McMahon, R. G., Walter, F., et al. 2012, ApJ, 751, L25

Wake, D. A., van Dokkum, P. G., \& Franx, M. 2012, ApJ, 751, L44

Walter, F., \& Carilli, C. 2008, Ap\&SS, 313, 313

Walter, F., Decarli, R., Carilli, C., et al. 2012, ApJ, 752, 93

Wang, R., Wagg, J., Carilli, C. L., et al. 2013, ApJ, 773, 44

Watson, D., Christensen, L., Knudsen, K. K., et al. 2015, Nature, 519,327

Willott, C. J., Bergeron, J., \& Omont, A. 2015, ApJ, 801, 123

Willott, C. J., Omont, A., \& Bergeron, J. 2013a, ApJ, 770, 13

Willott, C. J., McLure, R. J., Hibon, P., et al. 2013b, AJ, 145, 4 